\title{
SÍNTESIS DE ARSENIATOS DE CALCIO (GUERINITA, HAIDINGERITA Y FARMACOLITA) MORFOLÓGICAMENTE SIMILARES A LOS ENCONTRADOS EN SUELOS CONTAMINADOS
}

\author{
Gerardo HERNÁNDEZ-BÁRCENAS ${ }^{1}$, Francisco CASTILLO ${ }^{2}$, Miguel ÁVALOS-BORJA ${ }^{3,4}$ y \\ Nadia Valentina MARTÍNEZ-VILLEGAS ${ }^{1 *}$
}

${ }^{1}$ División de Geociencias Aplicadas, Instituto Potosino de Investigación Científica y Tecnológica. Camino a la Presa San José 2055, Colonia Lomas, 4ta. sección, San Luis Potosí, San Luis Potosí, México, C. P. 78216

${ }^{2}$ Instituto de Geología, Universidad Autónoma de San Luis Potosí. Avenida Dr. Manuel Nava 5, Zona Universitaria, San Luis Potosí, San Luis Potosí, México, C. P. 78240

${ }^{3}$ División de Materiales Avanzados, Instituto Potosino de Investigación Científica y Tecnológica. Camino a la Presa San José 2055, Colonia Lomas, 4ta. sección, San Luis Potosí, San Luis Potosí, México, C. P. 78216

${ }^{4}$ Centro de Nanociencias y Nanotecnología, Universidad Nacional Autónoma de México. Carretera TijuanaEnsenada, kilómetro 107, Ensenada, Baja California, México, C.P. 22800

*Autor para correspondencia: nadia.martinez@ipicyt.edu.mx

(Recibido octubre 2015; aceptado junio 2016)

Palabras clave: arsénico, arseniatos sintéticos, hábito cristalino, difracción de rayos X, microscopía electrónica de barrido, precipitación

\section{RESUMEN}

En el acuífero somero de Matehuala y Cerrito Blanco, en el estado de San Luis Potosí, México, se han reportado concentraciones de hasta $158 \mathrm{mg} / \mathrm{L}$ de arsénico cuya movilidad ha podido ser representada por la disolución de $\mathrm{Ca}_{5} \mathrm{H}_{2}\left(\mathrm{AsO}_{4}\right)_{4} \cdot n \mathrm{H}_{2} \mathrm{O}$, según modelaciones hidrogeoquímicas. Con la finalidad de probar la posible precipitación de este arseniato de calcio, en este estudio se llevaron a cabo experimentos de laboratorio de precipitación de arseniatos de calcio a $\mathrm{pH}$ y relación molar $\mathrm{Ca} / \mathrm{As}$ similares a las encontradas en el acuífero somero de Matehuala y Cerrito Blanco. Los experimentos de precipitación de arseniatos de calcio se llevaron a cabo en dos síntesis. Para la síntesis 1, se mezclaron soluciones $85.9 \mathrm{mM}$ de $\mathrm{Na}_{2} \mathrm{HAsO}_{4} \cdot 7 \mathrm{H}_{2} \mathrm{O}$ con suspensiones $0.93 \%(\mathrm{~m} / \mathrm{v})$ de $\mathrm{Ca}(\mathrm{OH})_{2}$. Para la síntesis 2 se mezclaron soluciones $114.4 \mathrm{mM}$ de $\mathrm{Na}_{2} \mathrm{HAsO}_{4} \cdot 7 \mathrm{H}_{2} \mathrm{O}$ con suspensiones $0.97 \%(\mathrm{~m} / \mathrm{v})$ de $\mathrm{Ca}(\mathrm{OH})_{2}$. En total, se prepararon 6 reactores que se almacenaron a temperatura ambiente por $500 \mathrm{~h}$. La temperatura, las concentraciones de arsénico, calcio y pH fueron medidos a lo largo del tiempo, mientras que los precipitados obtenidos fueron identificados por análisis de difracción de rayos X (DRX) y observados por microscopía electrónica de barrido (MEB). Los resultados de DRX muestran la precipitación de un arseniato de calcio [guerinita, $\mathrm{Ca}_{5} \mathrm{H}_{2}\left(\mathrm{AsO}_{4}\right)_{4} \cdot 9 \mathrm{H}_{2} \mathrm{O}$ ] con una estequiometría similar a la obtenida por modelación hidrogeoquímica $\left[\mathrm{Ca}_{5} \mathrm{H}_{2}\left(\mathrm{AsO}_{4}\right)_{4} \cdot n \mathrm{H}_{2} \mathrm{O}\right]$. Adicionalmente, se obtuvieron otros dos arseniatos de calcio llamados haidingerita $\left(\mathrm{CaHAsO}_{4} \cdot \mathrm{H}_{2} \mathrm{O}\right)$ y farmacolita $\left(\mathrm{CaHAsO}_{4} \cdot 2 \mathrm{H}_{2} \mathrm{O}\right)$. La observación de los precipitados obtenidos por MEB permitió la identificación de hábitos laminares, laminares aplanados y aciculares que se asociaron a haidingerita, guerinita y farmacolita, respectivamente. La morfología de los arseniatos de calcio precipitados en este estudio coincide con la observada en arseniatos de calcio encontrados en suelos contaminados en Matehuala. Los resultados de este estudio aportan al reconocimiento morfológico de arseniatos de calcio presentes en el ambiente. 
Key words: arsenic, synthetic arsenates, crystal habit, X-ray diffraction, scanning electron microscopy, precipitation

\begin{abstract}
Total dissolved arsenic concentrations up to $158 \mathrm{mg} / \mathrm{L}$ have been reported in the Matehuala and Cerrito Blanco aquifer in San Luis Potosi, Mexico from the dissolution of $\mathrm{Ca}_{5} \mathrm{H}_{2}\left(\mathrm{AsO}_{4}\right)_{4} \cdot n \mathrm{H}_{2} \mathrm{O}$, as determined by hydrogeochemical modelling. In order to test the precipitation of this calcium arsenate, in this study, calcium arsenates were synthesized at similar $\mathrm{pH}$ and $\mathrm{Ca} / \mathrm{As}$ aquifer conditions. Precipitation experiments were carried out in two synthesis. For synthesis 1 , calcium arsenates were prepared by mixing $85.9 \mathrm{mM} \mathrm{Na}_{2} \mathrm{HAsO}_{4} \cdot 7 \mathrm{H}_{2} \mathrm{O}$ solutions with slurries of $0.93 \%(\mathrm{w} / \mathrm{v}) \mathrm{Ca}(\mathrm{OH})_{2}$. For synthesis 2 , calcium arsenates were prepared by mixing $114.4 \mathrm{mM} \mathrm{Na} 2 \mathrm{HAsO}_{4} \cdot 7 \mathrm{H}_{2} \mathrm{O}$ solutions with slurries of $0.97 \%(\mathrm{w} / \mathrm{v}) \mathrm{Ca}(\mathrm{OH})_{2}$. A total of six reactors were prepared, they were stored at room temperature for $500 \mathrm{~h}$. Temperature, arsenic, calcium concentrations and $\mathrm{pH}$ were measured over time while the precipitates were identified by X-ray diffraction analyses and observed by scanning electron microscopy. According to X-ray diffraction results and the experimental conditions, a calcium arsenate, namely guerinite $\left[\mathrm{Ca}_{5} \mathrm{H}_{2}\left(\mathrm{AsO}_{4}\right)_{4} \cdot 9 \mathrm{H}_{2} \mathrm{O}\right]$ with the predicted stoichiometry, precipitated. Additionally, other two calcium arsenate mineral phases, namely haidingerite $\left(\mathrm{CaHAsO}_{4} \cdot \mathrm{H}_{2} \mathrm{O}\right)$ and pharmacolite $\left(\mathrm{CaHAsO}_{4} \cdot 2 \mathrm{H}_{2} \mathrm{O}\right)$ precipitated. The observation of the calcium arsenates precipitates allowed the identification of laminar, platty, and acicular habits associated to haidingerite, guerinite and pharmacolite, respectively. The morphology of the calcium arsenates precipitates in this study agrees with the morphology observed in calcium arsenates found in contaminated soils in Matehuala. Results from this study contribute to the morphological identification of calcium arsenates in the environment.
\end{abstract}

\section{INTRODUCCIÓN}

El arsénico es un elemento ubicuo, que se encuentra en la atmósfera, el suelo, rocas, aguas naturales e incluso en organismos vivos (Smedley y Kinniburgh 2002). Debido a su toxicidad, es reconocido globalmente como una de las amenazas ambientales más grandes (Ravenscroft et al. 2009)

Adicionalmente, el arsénico también se encuentra en el ambiente por actividad antropogénica, dentro de la cual podemos mencionar a la metalurgia. La adición de cal a residuos industriales metalúrgicos, entre otros, ha sido utilizada de hecho, como técnica de estabilización para incrementar el $\mathrm{pH}$ de dichos residuos. A través de esta técnica se pueden alcanzar valores de remoción de arsénico incluso superiores al $95 \%$ (Zhu et al. 2006), dando origen a la formación de arseniatos de calcio de, presuntamente baja solubilidad (Bothe y Brown 1999a). Por el contrario, se ha observado que dichos arseniatos de calcio, poseen una alta solubilidad en el ambiente (MartínezVillegas et al. 2013). Las investigaciones acerca de la estabilización de arsénico utilizando $\mathrm{CaO}$ y $\mathrm{Ca}(\mathrm{OH})_{2}$ han propuesto la formación de fases sólidas de estequiometría, grados de hidratación, estabilidad y estructura cristalina variadas (Nishimura y Robins 1998, Bothe y Brown 1999a, 1999b, 2002, Zhu et al. 2006). De forma general, las fases sólidas formadas incluyen hidratos de hidroxiarseniatos de calcio $\left[\mathrm{Ca}_{4}(\mathrm{OH})_{2}(\mathrm{AsO} 4)_{2} \cdot 4 \mathrm{H}_{2} \mathrm{O}\right.$ y Ca $\left.5\left(\mathrm{AsO}_{4}\right)_{3} \mathrm{OH}\right]$, hidratos de arseniatos de calcio $\left[\mathrm{Ca}_{3}\left(\mathrm{AsO}_{4}\right)_{2} \cdot n \mathrm{H}_{2} \mathrm{O}\right.$ donde $n=$ 2.25, 3.0, 3.67, 4.0 y 4.25], arseniatos diprotonados de calcio [isómeros de $\mathrm{Ca}_{5} \mathrm{H}_{2}\left(\mathrm{AsO}_{4}\right)_{4} .9 \mathrm{HO}$ como ferrarisita y guerinita] y arseniatos monoprotonados de calcio [ $\mathrm{CaHAsO}_{4} \cdot n \mathrm{H}_{2} \mathrm{O}$ donde $n=1$ y 2] (Bothe y Brown 1999a, 1999b, Zhu et al. 2006). La estructura de los precipitados de arseniato de calcio depende del pH y de la relación $\mathrm{Ca}$ /As (Zhu et al. 2006). La relación $\mathrm{Ca} / \mathrm{As}$ establece la disponibilidad de ion calcio mientras que el $\mathrm{pH}$ determina la especiación del arsénico en la fase acuosa y por lo tanto, el tipo de precipitado formado.

En ambientes naturales e impactados, la identificación inequívoca de arseniatos de calcio ha demostrado, sin embargo, ser muy difícil de llevar a cabo (Ondrus et al. 1997, Donahue y Hendry 2003, Onac et al. 2007, Pantuzzo y Ciminelli 2010, MartínezVillegas et al. 2013, Castillo et al. 2015) debido a la presencia de variados y diferentes arseniatos de calcio en muestras compuestas principalmente por otros 
minerales primarios (Ondrus et al. 1997, Donahue y Hendry 2003, Onac et al. 2007, Pantuzzo y Ciminelli 2010, Martínez-Villegas et al. 2013, Castillo et al. 2015). Aunado a la falta de reproducibilidad y ambigüedad de patrones de difracción de fases puras de arseniatos de calcio que no empatan con alguna fase conocida (Myneni et al. 1997, Bothe y Brown 1999a, 1999b). Por ejemplo, para el acuífero somero de Matehuala y Cerrito Blanco, en el estado de San Luis Potosí, México, se han reportado concentraciones de hasta $158 \mathrm{mg} / \mathrm{L}$ de arsénico disuelto derivado de ciclos de disolución y precipitación de arseniatos de calcio cuya modelación hidrogeoquímica predice el control de la movilidad de arsénico por un arseniato de calcio de estequiometria $\mathrm{Ca}_{5} \mathrm{H}_{2}\left(\mathrm{AsO}_{4}\right)_{4} n \mathrm{H}_{2} \mathrm{O}$ (Martínez-Villegas et al. 2013). Con la finalidad de comprender mejor el control de la movilidad del arsénico en dicho acuífero, en este estudio se sintetizaron arseniatos de calcio a un $\mathrm{pH}$ y una relación molar $\mathrm{Ca} /$ As similares a las que se encuentran en el acuífero contaminado de Matehuala y Cerrito Blanco. Los precipitados obtenidos se analizaron por difracción de rayos X para su identificación y por microscopia electrónica de barrido para observar su morfología.

\section{MATERIALES Y MÉTODOS}

\section{Síntesis de los arseniatos de calcio}

Un total de dos experimentos de síntesis fueron llevados a cabo por triplicado mezclando soluciones de $\mathrm{Na}_{2} \mathrm{HAsO}_{4} \cdot 7 \mathrm{H}_{2} \mathrm{O}$ con suspensiones de $\mathrm{Ca}(\mathrm{OH})_{2}$. Para la primer síntesis se mezclaron $50 \mathrm{~mL}$ de $\mathrm{Na}_{2} \mathrm{HAsO}_{4} .7 \mathrm{H}_{2} \mathrm{O}(86 \mathrm{mM})$ y $50 \mathrm{~mL}$ de $0.93 \%(\mathrm{~m} / \mathrm{v})$ de $\mathrm{Ca}(\mathrm{OH})_{2}$ en matraces Erlenmeyer de $125 \mathrm{~mL}$ a temperatura ambiente. Posteriormente se ajustó el pH a 7.0 utilizando $\mathrm{HNO}_{3}$ concentrado. Los matraces se identificaron como S1-1, S1-2 y S1-3, donde $\mathrm{S} 1$ se refiere a la síntesis 1 y el 1,2,3 a las réplicas. Para la segunda síntesis se prepararon $50 \mathrm{~mL}$ de $\mathrm{Na}_{2} \mathrm{HAsO}_{4} .7 \mathrm{H}_{2} \mathrm{O}(114 \mathrm{mM})$ y $50 \mathrm{~mL}$ de $0.97 \%(\mathrm{~m} / \mathrm{v})$ de $\mathrm{Ca}(\mathrm{OH})_{2}$ a pH 7.0 cada una. Posteriormente, las soluciones se mezclaron en matraces Erlenmeyer de $125 \mathrm{~mL}$ y los matraces se identificaron como S2-1, $\mathrm{S} 2-2$ y S2-3, donde $\mathrm{S} 2$ se refiere a síntesis 2 y el 1 , 2,3 a las réplicas. La diferencia entre la síntesis 1 y la síntesis 2 radica en el ajuste de $\mathrm{pH}$ a 7.0 después y antes de la mezcla, respectivamente y en la relación $\mathrm{Ca} / \mathrm{As}$. Mientras que el ajuste del $\mathrm{pH}$ a 7.0 después de la mezcla permitió una relación $\mathrm{Ca} / \mathrm{As}$ $=1.04$, el ajuste de $\mathrm{pH}$ antes de la mezcla permitió alcanzar una relación $\mathrm{Ca} / \mathrm{As}=1.25$, siendo esta última similar a la relación del arseniato de calcio que reproduce el comportamiento del arsénico en el acuífero contaminado de Matehuala y Cerrito Blanco, según Martínez-Villegas et al. (2013). A lo largo del tiempo, la temperatura y el $\mathrm{pH}$ fueron monitoreados utilizando un termómetro y un potenciómetro de mesa Thermo Scientific Orion Versa Star. Adicionalmente, cada $0,2,4,16,32,64,128,256$ y 500 h se recolectaron muestras de $1 \mathrm{~mL}$ de suspensión de cada reactor utilizando jeringas desechables. Cada muestra fue filtrada a través de membranas de $0.45 \mu \mathrm{m}$ de diámetro de poro, diluida a $50 \mathrm{~mL}$, estabilizada con $\mathrm{HNO}_{3}$ hasta alcanzar un $\mathrm{pH}<2$ y almacenada a $4{ }^{\circ} \mathrm{C}$ hasta su análisis por espectroscopía de emisión óptica de plasma acoplado inductivamente (ICP-OES) para calcio y arsénico utilizando un espectrofotómetro Varian 730.

\section{Identificación de los arseniatos de calcio}

La identificación de los precipitados obtenidos en este trabajo se llevó a cabo por difracción de rayos $\mathrm{X}$ (DRX). Al final de las 500 h, las fases sólidas de cada matraz fueron decantadas y secadas a temperatura ambiente. Cada muestra fue homogenizada y compactada en un soporte de vidrio con una superficie de $1.5 \mathrm{~cm}^{2}$ y analizada en un intervalo $2 \theta$ de 5 a $90^{\circ}$ con una velocidad de paso de $0.02 \%$, a escaneo continuo, utilizando un difractómetro de rayos X Bruker D8 ADVANCE, equipado con una fuente de $\mathrm{Cu} \mathrm{K} \alpha$, con ventana de divergencia $2 \mathrm{~mm}$, rendija antidispersión de $2 \mathrm{~mm}$, rendija del detector $0.2 \mathrm{~mm}$ y filtros de níquel. Esta forma de preparación y análisis permite que la muestra tenga todas las orientaciones posibles de los cristales y por tanto todas las reflexiones se pueden medir en un único patrón de difracción. Los difractogramas obtenidos de las fases precipitadas se procesaron utilizando el paquete X'pert HighScore Plus con la finalidad de identificar las fases presentes y compararlas con la base de datos $\mathrm{PDF}^{+}$del Centro Internacional de Datos de Difracción (ICDD, por sus siglas en inglés).

\section{Morfología de los arseniatos de calcio}

Una muestra de aproximadamente $0.5 \mathrm{~mL}$ de suspensión del fondo de cada matraz fue recolectada cada $0,2,4,16,32,64,128,256$ y 500 h utilizando una pipeta de transferencia Pasteur con la finalidad de observar la morfología de los agregados que se iban precipitando a lo largo del tiempo y, posteriormente, asociar los hábitos cristalinos observados a las fases precipitadas. Después de la colecta, cada muestra se colocó sobre una oblea de silicio de aproximadamente $1 \mathrm{~cm}^{2}$ y se analizó utilizando un microscopio electrónico de barrido FEI Quanta 200 acoplado a un 
sistema de energía dispersiva (EDS). Los análisis de microscopía electrónica de barrido (MEB) se realizaron con un detector de electrones retrodispersados en condiciones de bajo vacío $(10-130 \mathrm{kPa})$ para evitar que la muestra presentara el típico efecto de carga de los materiales no conductores, efecto indeseable que impediría la obtención de imágenes de buena calidad. Adicionalmente, las micrografías de este estudio se compararon con imágenes de MEB correspondientes a muestras de suelos de Martínez-Villegas et al. (2013), con la finalidad de identificar similitudes en los hábitos cristalinos de arseniatos de calcio entre ambos estudios.

\section{RESULTADOS}

\section{Síntesis de los arseniatos de calcio}

La figura 1 muestra la evolución de la temperatura, el $\mathrm{pH}$, las concentraciones de arsénico y calcio, así como la relación $\mathrm{Ca} / \mathrm{As}$ a lo largo del tiempo para la síntesis 1 y 2 . Las síntesis se llevaron a cabo a temperatura ambiente, con una temperatura media de $22 \pm 2{ }^{\circ} \mathrm{C}$ para la síntesis 1 y de $23 \pm 1{ }^{\circ} \mathrm{C}$ para la 2. La temperatura mínima para la síntesis 1 fue de $19{ }^{\circ} \mathrm{C}$ (Fig. 1a) y $20{ }^{\circ} \mathrm{C}$ para la 2 (Fig. 1a). La temperatura máxima registrada fue de $26{ }^{\circ} \mathrm{C}$ para ambas síntesis (Fig. 1a). Inicialmente, el $\mathrm{pH}$ de la síntesis 1 fue de $12.30 \pm 0.11$ (Fig. 1b), mismo que disminuyó a $6.99 \pm 0.03$ con la adición de $\mathrm{HNO}_{3}$. Después del ajuste, el $\mathrm{pH}$ de la síntesis 1 se mantuvo constante (Fig. 1b). A las 500 h, el pH de la síntesis 1 fue de $6.67 \pm 0.03$ (Fig. 1b). En lo que se refiere a la concentración de arsénico total, ésta disminuyó a lo largo del tiempo. A las 500 h, disminuyó de 85.9 a $35.13 \pm 1.71 \mathrm{mmol} / \mathrm{L}$ para la síntesis 1 y de 114.4 a $36.44 \pm 1.23 \mathrm{mmol} / \mathrm{L}$ para la síntesis 2 (Fig. 1c). Por otro lado, la concentración inicial de calcio en solución fue de $89.2 \mathrm{mmol} / \mathrm{L}$ en la síntesis $1 \mathrm{y}$ $128.7 \mathrm{mmol} / \mathrm{L}$ en la 2 (Fig. 1d). Al final del experimento, la concentración de calcio total en solución disminuyó a $46.56 \pm 2.35 \mathrm{mmol} / \mathrm{L}$ para la síntesis 1 y a $41.17 \pm 0.90 \mathrm{mmol} / \mathrm{L}$ para la 2 (Fig. 1d). Las disminuciones en las concentraciones de arsénico y calcio en la solución coincidieron con la precipitación de arseniatos de calcio, misma que fue observable a simple vista desde aproximadamente las $2 \mathrm{~h}$ del comienzo del experimento (ver secciones siguientes). La tendencia asintótica del $\mathrm{pH}$ y las concentraciones de arsénico y calcio se interpretaron como el alcance del equilibrio de la reacción. Dicha tendencia se observó a partir de las 16 h de reacción (Fig. 1b-d).
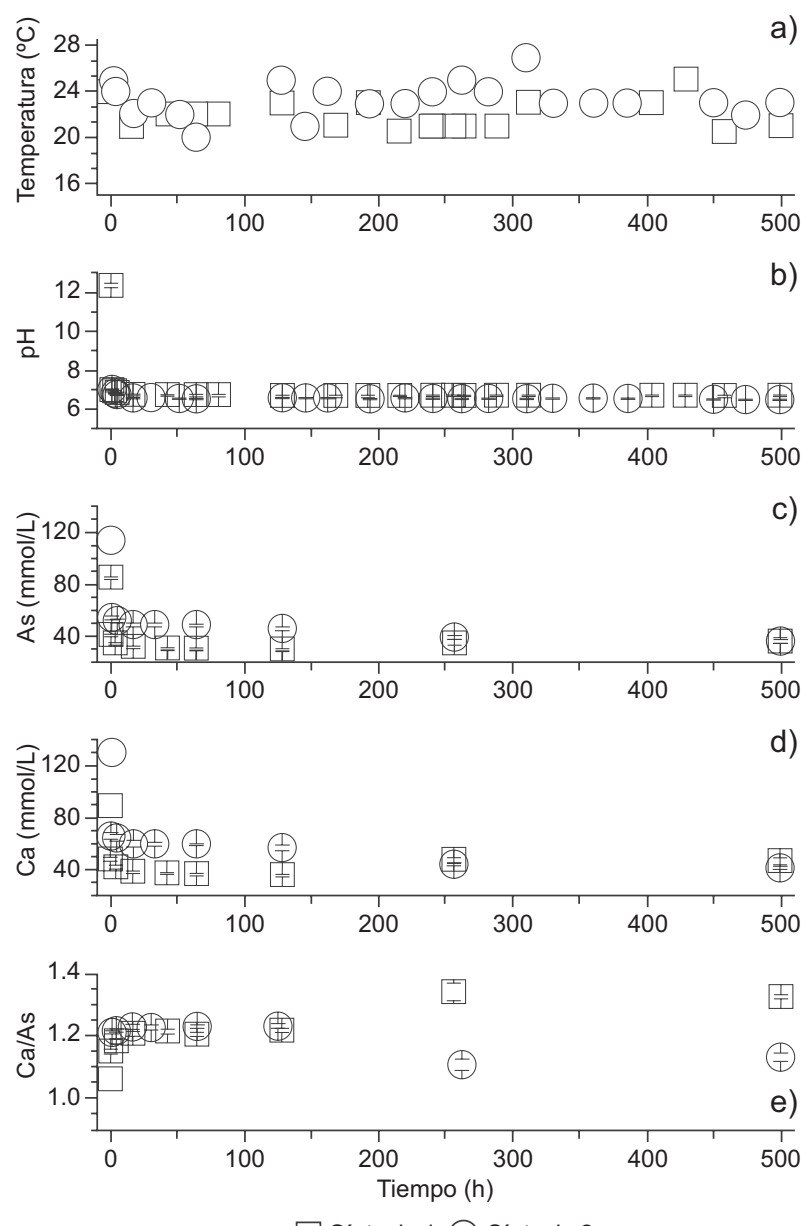

$\square$ Síntesis $1 \bigcirc$ Síntesis 2

Fig. 1. Valores de a) temperatura, b) $\mathrm{pH}$, c) concentraciones de $\mathrm{As}, \mathrm{d}$ ) concentraciones de $\mathrm{Ca}$ y e) relación $\mathrm{Ca} / \mathrm{As}$ a lo largo del tiempo. Las barras de error representan la desviación estándar de los datos. Los datos de temperatura, así como los de $\mathrm{As}$ y $\mathrm{Ca}$ a $\mathrm{t}=0 \mathrm{~h}$ se midieron para sólo una réplica por lo que no cuentan con desviaciones estándar

Los experimentos se detuvieron a las $500 \mathrm{~h}$ (Fig. 1b-d). En lo que se refiere a la relación molar $\mathrm{Ca} /$ As, para la síntesis 1 , dicha relación aumentó de 1.06 a 1.32 en el equilibrio (Fig. 1e), mientras que para la síntesis 2 , la relación $\mathrm{Ca} / \mathrm{As}$ comenzó en 1.20 y finalizó con un valor de 1.12 en el equilibrio (Fig. 1e). Es interesante observar que a un mayor $\mathrm{pH}$ inicial, se obtiene una relación $\mathrm{Ca} / \mathrm{As}$ más baja como resultado de una menor disponibilidad de calcio a $\mathrm{pH}$ alto y viceversa (Fig. 1b, d y e). Adicionalmente, que las condiciones iniciales de $\mathrm{pH}$ y por consecuencia, de disponibilidad de calcio, parecen tener un impacto en la relación $\mathrm{Ca} / \mathrm{As}$ al término del experimento, independientemente del valor final del $\mathrm{pH}$ al concluir las síntesis. 


\section{Identificación de arseniatos de calcio}

La figura 2 muestra los difractogramas de la síntesis 1 , dentro de los picos obtenidos. Los situados en las posiciones $2 \theta=6.2,7.5,10.02$ y 29.4 corresponden a las reflexiones principales de guerinita, mientras que los picos ubicados en las posiciones $2 \theta=10.9,30.3,27.9,16.8$ y 17.01 corresponden a haidingerita. Como puede observarse, dichas posiciones explican por completo los difractogramas de las muestras de la síntesis 1 (Fig. 2), por lo que a estas condiciones ( $\mathrm{pH} 6.67$ y una relación $\mathrm{Ca} / \mathrm{As}=1.32$ ) se concluyó que los arseniatos de calcio que precipitaron fueron guerinita y haidingerita. En el caso de la síntesis 2 se identificaron picos en las posiciones $2 \theta$ $=6.2,7.5,10.02,29.4$ correspondientes a guerinita $\mathrm{y}$ en las posiciones $2 \theta=10.9,30.3,27.9,16.8,17.01$ correspondientes a las reflexiones principales de haidingerita, además las posiciones $2 \theta=11.4,20.7$, 28.8 y 29.4 pertenecieron a farmacolita (Fig. 3).
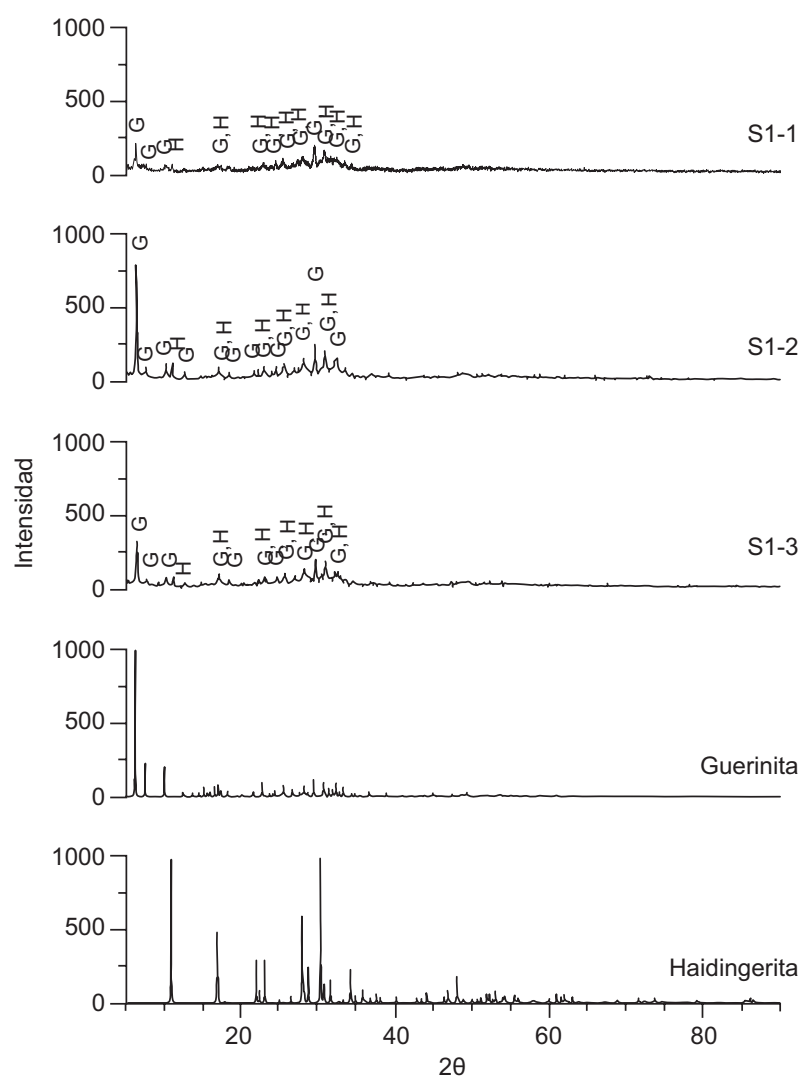

Fig. 2. Difractogramas de rayos $X$ de la síntesis 1 mostrando que los picos experimentales empatan con los difractogramas simulados de guerinita (Tarjeta PDF Núm. 01-070-1034) y haidingerita (Tarjetas PDF Núm. 01-085-1384, 01075-0899, 01-070-1581 para S1-1, S1-2 y S1-3, respectivamente) de acuerdo con la base de datos PDF4 ${ }^{+}$del Centro Internacional para Datos de Difracción
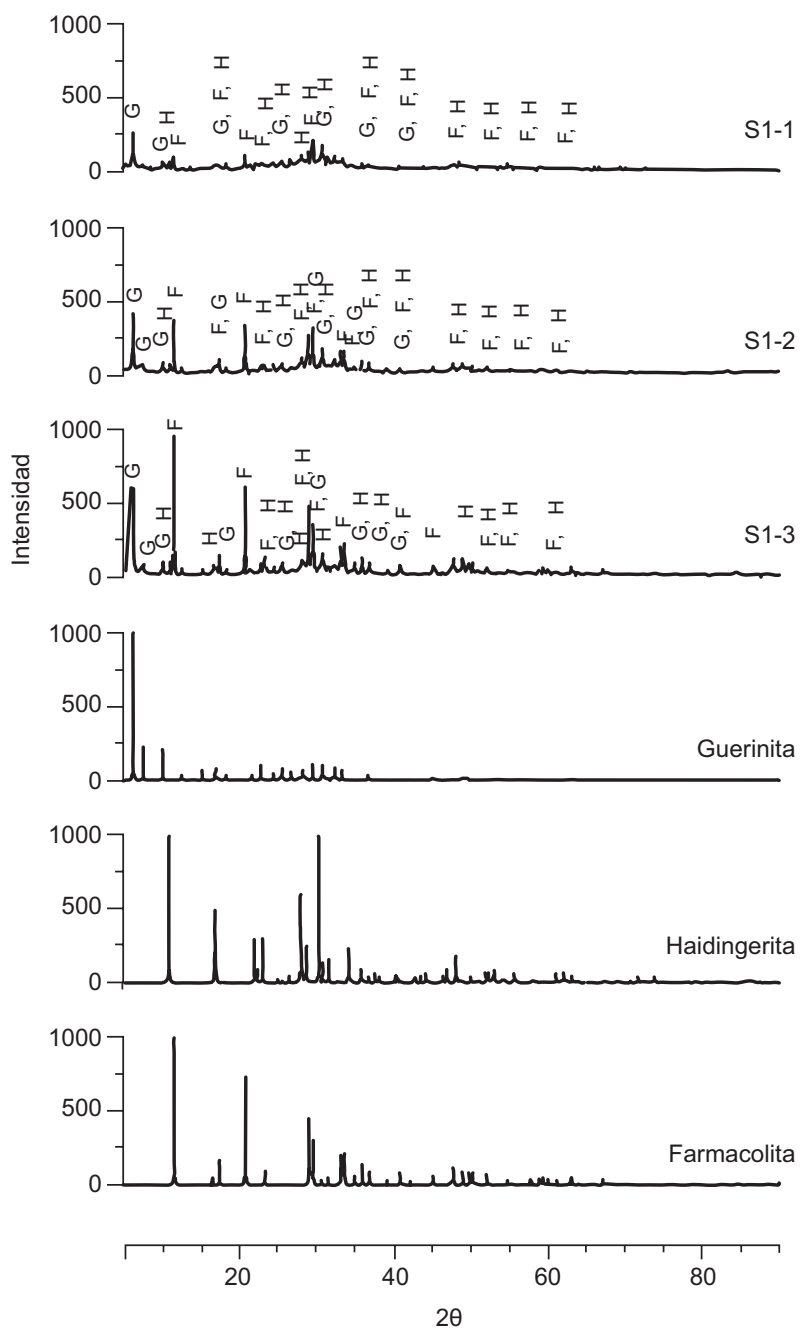

Fig. 3. Difractogramas de rayos $\mathrm{X}$ de la síntesis 2 mostrando que los picos experimentales empatan con los difractogramas simulados de guerinita (Tarjeta PDF Núm. 01-070-1034), haidingerita (Tarjeta PDF Núm. 01-075-0899) y farmacolita (Tarjeta PDF Núm. 01-074-0602), de acuerdo con la base de datos $\mathrm{PDF}^{+}$del Centro Internacional para Datos de Difracción

Como puede observarse, dichos picos explican por completo los difractogramas de las muestras de la síntesis 2 (Fig. 3), por lo que se concluyó que a estas condiciones $(\mathrm{pH} 6.50$ y una relación $\mathrm{Ca} / \mathrm{As}=$ 1.12) los arseniatos de calcio que precipitaron fueron guerinita, haidingerita y farmacolita. Es interesante observar que la precipitación de farmacolita se presentó sólo en los experimentos con menor $\mathrm{pH}$ inicial.

\section{Morfología de los arseniatos de calcio}

Los precipitados obtenidos, observables a simple vista a partir de $\sim 2 \mathrm{~h}$ del experimento, presentaron morfologías características de arseniatos de calcio 
(Ondrus et al. 1997, Nishimura y Robins 1998, Jiménez et al. 2006, Schorrn 2006, Zhu et al. 2006, Rodríguez-Blanco et al. 2007, Rodríguez et al. 2008). Las morfologías observadas corresponden a hábitos laminares y laminares aplanados cuyos agregados presentaron estructuras radiales y/o fibrosas (Fig. 4-6). Además, se observaron también hábitos aciculares (Fig. 7).

Dentro de las morfologías laminares, se observaron laminas (Fig. 4a) con bordes angulares que pueden terminar en forma aplanada (Fig. $4 \mathrm{~b}$ y c) o en vértices afilados en los extremos (Fig. 4d). Estas morfologías se presentaron a todos los tiempos de muestreo y en todos los reactores sin una tendencia cronológica clara. No obstante, se propone que existe una tendencia de evolución que tiene como punto de partida una lámina (Fig. 4a), que puede agregarse con otras láminas paralelamente (Fig. 4 b y c) o con ángulos variables entre sus planos (Fig. $4 \mathbf{d}$ y e), unidas en ambos casos en el centro de las láminas (Fig. 4b-h)
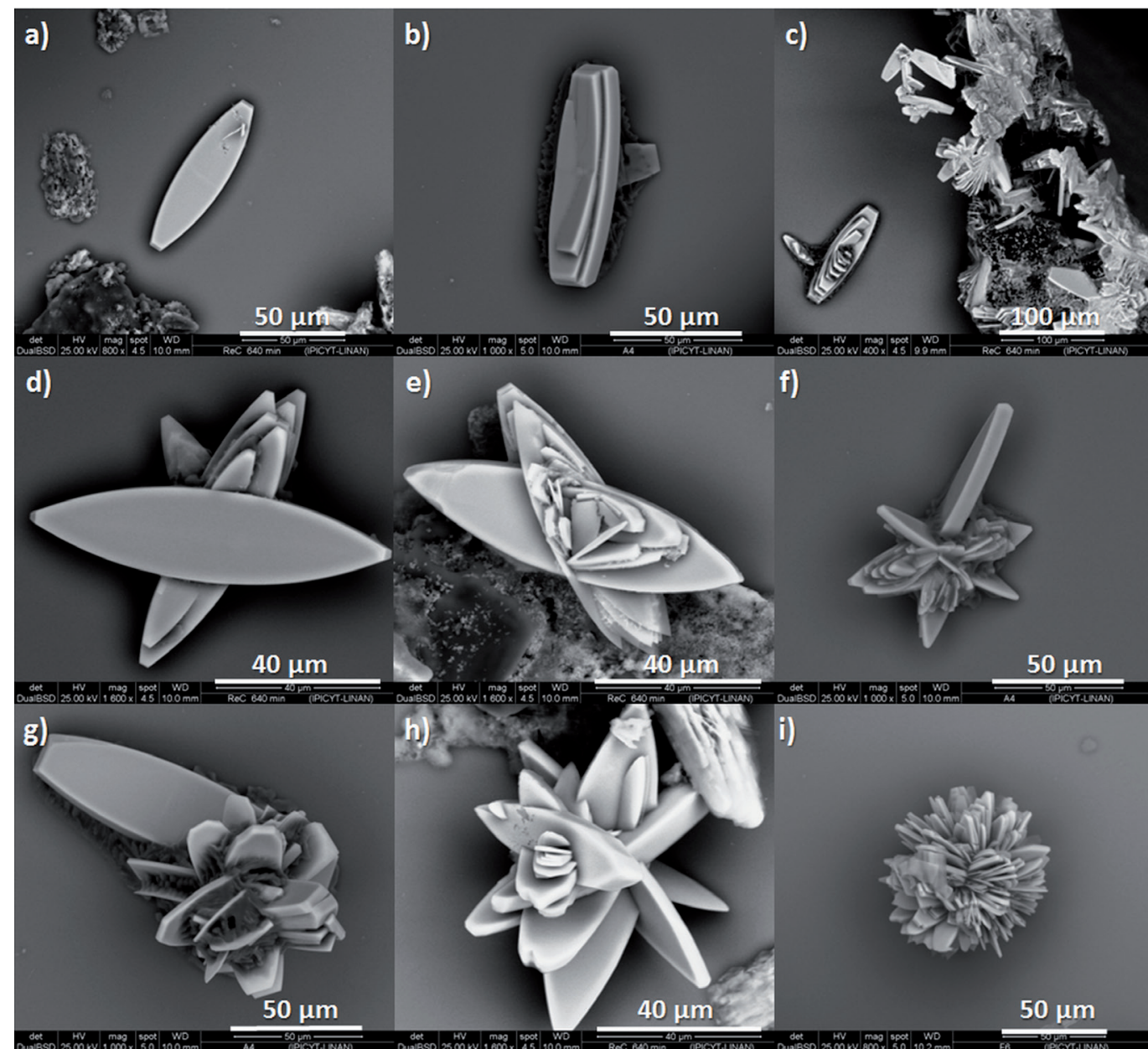

Fig. 4. Láminas de haidingerita que parecen agregarse una sobre otra en el centro para dar origen a estructuras radiales: a) lámina observada en la muestra $\mathrm{S} 1-1$ recolectada al tiempo $40 \mathrm{~h}, \mathrm{~b}$ ) láminas agregadas paralelamente observadas en la muestra S1-1 recolectada al tiempo $40 \mathrm{~h}$, c) láminas agregadas paralelamente en el centro que aparentan doblarse y/o exfoliarse y, a la derecha, cúmulos de estructuras del mismo tipo, observadas en la muestra S1-1 recolectada al tiempo $40 \mathrm{~h}$, d) láminas con vértices afilados agregadas con diferente orientación una sobre otra, observadas en la muestra S1-1 recolectada al tiempo $40 \mathrm{~h}, \mathrm{e}$ ) estructura de láminas con vértices afilados y bordes angulares achatados observada en la muestra S1-1 recolectada al tiempo $40 \mathrm{~h}, \mathrm{f}$ ) estructura radial de láminas con vértices afilados observada en la muestra S1-1 recolectada al tiempo $40 \mathrm{~h}, \mathrm{~g}$ ) agregado de láminas con bordes mayoritariamente achatados en arreglo radial observado en la muestra S1-1 recolectada al tiempo $40 \mathrm{~h}, \mathrm{~h}$ ) agregado radial de láminas con bordes achatados y vértices afilados observada en la muestra S1-1 recolectada al tiempo $40 \mathrm{~h}$ e i) estructura radial formada de láminas agregadas radialmente observada en la muestra S1-1 recolectada al tiempo $500 \mathrm{~h}$ 


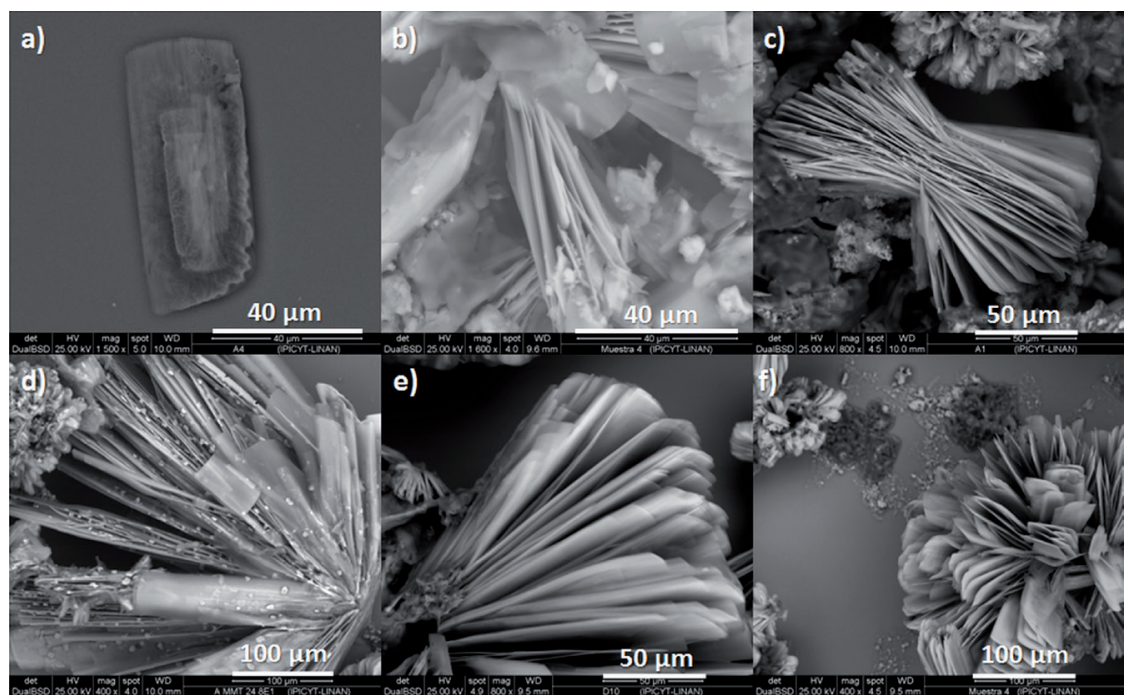

Fig. 5. Guerinita en forma de láminas delgadas que parecen agregarse una sobre otra en el centro para dar origen a estructuras laminares. a) vista en planta de estructuras laminares delgadas observadas en la muestra S1-1 recolectada al tiempo $40 \mathrm{~h}$, b) vista lateral de láminas delgadas agregadas en estructuras laminares observadas en la muestra S1-1 recolectada al tiempo $4 \mathrm{~h}, \mathrm{c}$ ) vista lateral de láminas delgadas agregadas paralelamente unidas en el centro que aparentan doblarse y/o exfoliarse dando origen a estructuras laminares con un mayor espacio entre láminas en los extremos exteriores; observadas en la muestra S1-1 recolectada al tiempo $2 \mathrm{~h}$, d) vista lateral de láminas delgadas agregadas paralelamente una sobre otra en una estructura laminar con pequeñas motas blancas (de arseniatos de calcio) depositadas sobre ellas observadas en la muestra S1-1 recolectada al tiempo $16 \mathrm{~h}, \mathrm{e}$ ) vista en planta de estructura laminar mostrando la presencia copiosa de láminas delgadas que parecen exfoliarse de las láminas principales; estructura observada en la muestra S1-1 recolectada al tiempo $500 \mathrm{~h}$ y f) estructura radial formada de láminas delgadas agregadas observada en la muestra S1-1 recolectada al tiempo $4 \mathrm{~h}$

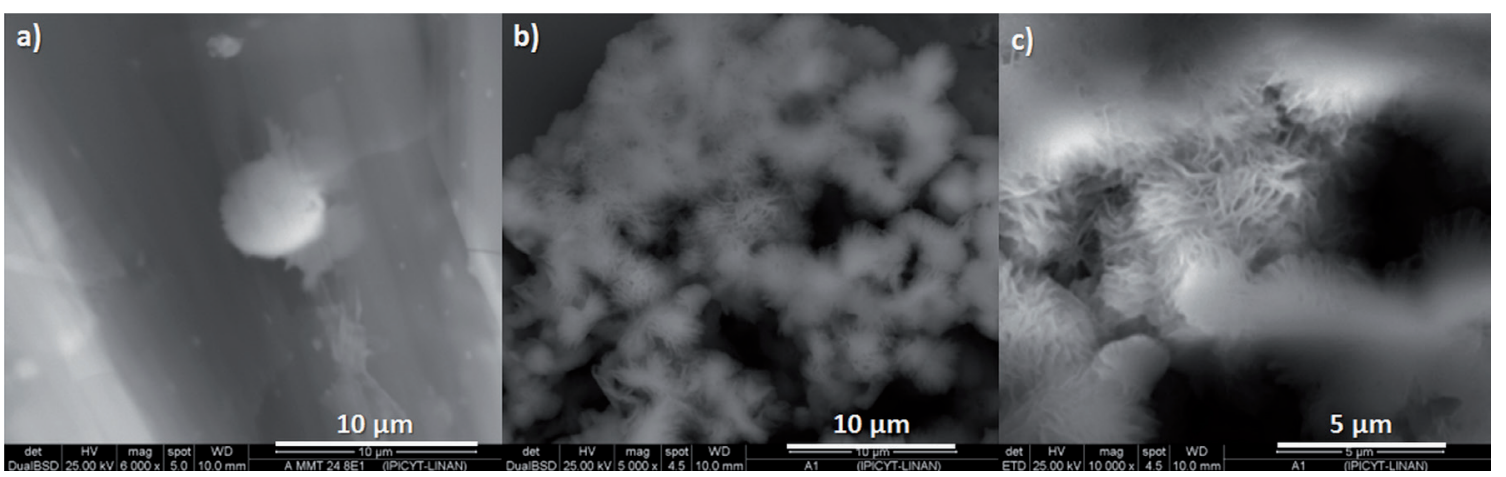

Fig. 6. Haidingerita en aspecto fibroso. a) espécimen de haidingerita en estructura esférica de aspecto fibroso observado en la muestra S1-1 recolectada al tiempo 16 h y comúnmente encontrada en los experimentos de síntesis de arseniatos de calcio (Fig. 5d), b) cúmulo fibroso de haidingerita observado en la muestra S1-1 recolectada al tiempo $2 \mathrm{~h}, \mathrm{c}$ ) acercamiento del cúmulo en $\mathrm{b}$ ), mostrando los pequeños filamentos que conforman las estructuras esféricas de haidingerita observadas en la muestra S1-1 recolectada al tiempo $2 \mathrm{~h}$

hasta la formación de estructuras radiales en arreglos casi esféricos (Fig. 4g-i). Dicha morfológica laminar y estructura radial se asoció con los hábitos cristalinos reportados en la literatura para haidingerita (Ondrus et al. 1997, MinDat 2016a).
Dentro de las morfologías laminares, también se observaron láminas delgadas agrupadas en estructuras foliares (Fig. 5a-b), unidas y compactas en el centro (Fig. 5c), con espacio entre láminas en el extremo exterior (Fig. 5c-e). De forma similar a las 


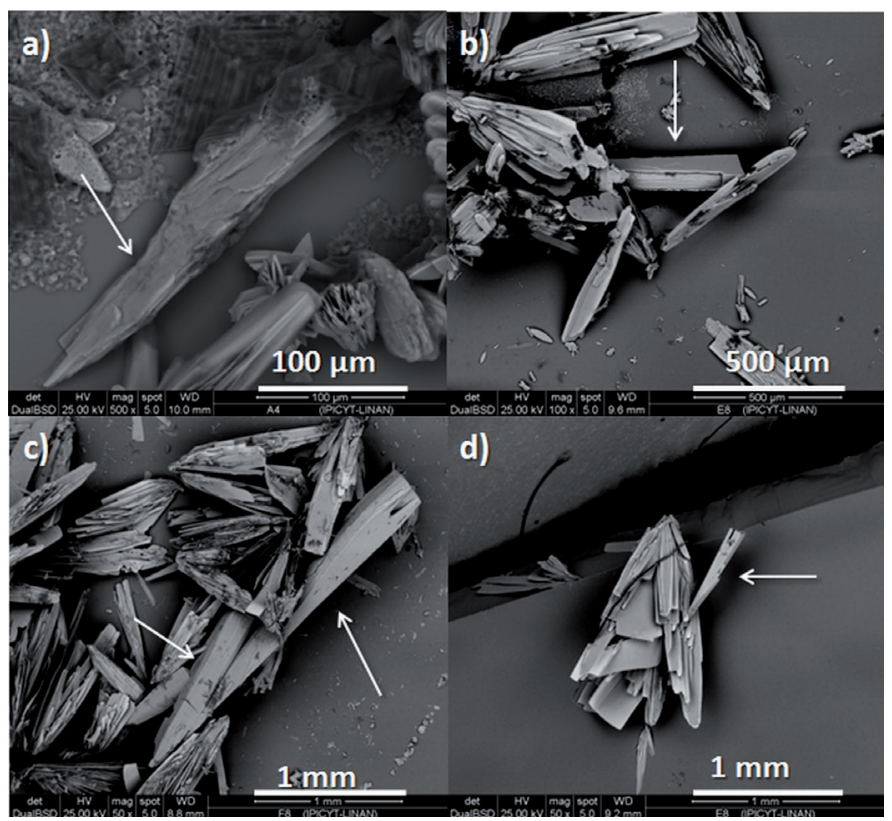

Fig. 7. Farmacolita en forma de agujas. a) farmacolita en forma de aguja observada en la muestra S1-1 recolectada al tiempo $40 \mathrm{~h}$, b) aguja observada en la muestra $S 2-2$ recolectada al tiempo 500 h, c) aguja observada en la muestra S2-3 recolectada al tiempo $500 \mathrm{~h}$ y d) aguja observada en la muestra S2-3 recolectada al tiempo $500 \mathrm{~h}$

láminas de haidingerita, se propone que éstas pueden agregarse una sobre otra (Fig. 5a y b), unirse en un punto en común (Fig. 5c-e) y dar paso a la formación de agregados foliados (Fig. 5c-e), los cuales parecen unirse a otros agregados. Al compartir el punto de unión, consiguen agruparse y formar en algunos casos, estructuras radiales que pueden llegar a ser esféricas (Fig. 5f). Dicha morfología laminar delgada se asoció con los hábitos cristalinos observados en la literatura para guerinita (Ondrus et al. 1997, MinDat 2016b).

Adicionalmente a las estructuras esféricas de haidingerita y guerinita, también se observaron formas esféricas, con tamaño de radio de $\sim 5 \mu \mathrm{m}$, aparentemente formadas por filamentos o fibras delgadas (Fig. 6). Dicho hábito fibroso ha sido asociado en la literatura a haidingerita y farmacolita (MinDat, 2016a, 2016c). Sin embargo, en estas muestras (S1), la difracción de rayos $\mathrm{X}$ indica sólo la presencia de haidingerita por lo que dicha morfología se asoció a haidingerita.

Por último, se observaron morfologías aciculares (es decir, en forma de aguja), las cuales presentaron puntas angulares en forma de prisma triangular y superficies irregulares con tamaños que varían entre $250 \mu \mathrm{m}$ y $1.6 \mathrm{~mm}$ (Fig. 7). Dichos hábitos aciculares han sido reportados para farmacolita (Ondrus et al.
1997, Jiménez et al. 2006, Rodríguez-Blanco et al. 2007, MinDat 2016c) y a su vez, han sido observados en hábitos cristalinos de agregados de arseniatos de calcio en suelos contaminados en Matehuala (Martínez-Villegas et. al. 2013). De hecho, las morfologías correspondientes a haidingerita, guerinita y farmacolita observadas en este estudio son similares a arseniatos de calcio observados en suelos contaminados recolectados durante la realización del estudio de Martínez-Villegas et al. (2013) (Fig. 8).

\section{DISCUSIÓN}

Las fases sintetizadas en este estudio, corresponden a arseniatos monoprotonados de calcio con una relación $\mathrm{Ca} / \mathrm{As}=1.0\left[\mathrm{CaHAsO}_{4} \cdot n \mathrm{H}_{2} \mathrm{O}\right]$ y arseniatos diprotonados de calcio con una relación $\mathrm{Ca} / \mathrm{As}=1.25$ $\left[\mathrm{Ca}_{5} \mathrm{H}_{2}\left(\mathrm{AsO}_{4}\right)_{4} . n \mathrm{H}_{2} \mathrm{O}\right]$ con distinto grado de hidratación (Cuadro I). En el caso de $\mathrm{CaHAsO}_{4} \cdot n \mathrm{H}_{2} \mathrm{O}$ con $n=1$ para haidingerita y $n=2$ para farmacolita. Para $\mathrm{Ca}_{5} \mathrm{H}_{2}\left(\mathrm{AsO}_{4}\right)_{4} \cdot n \mathrm{H}_{2} \mathrm{O}$ con $n=9$ para guerinita. Las fases identificadas en este trabajo concuerdan con las fases identificadas por Castillo et al. (2015), quien identificó guerinita, haidingerita y farmacolita en muestras de suelos contaminados con arseniatos de calcio recolectados en Matehuala (Castillo et al. 2015). 


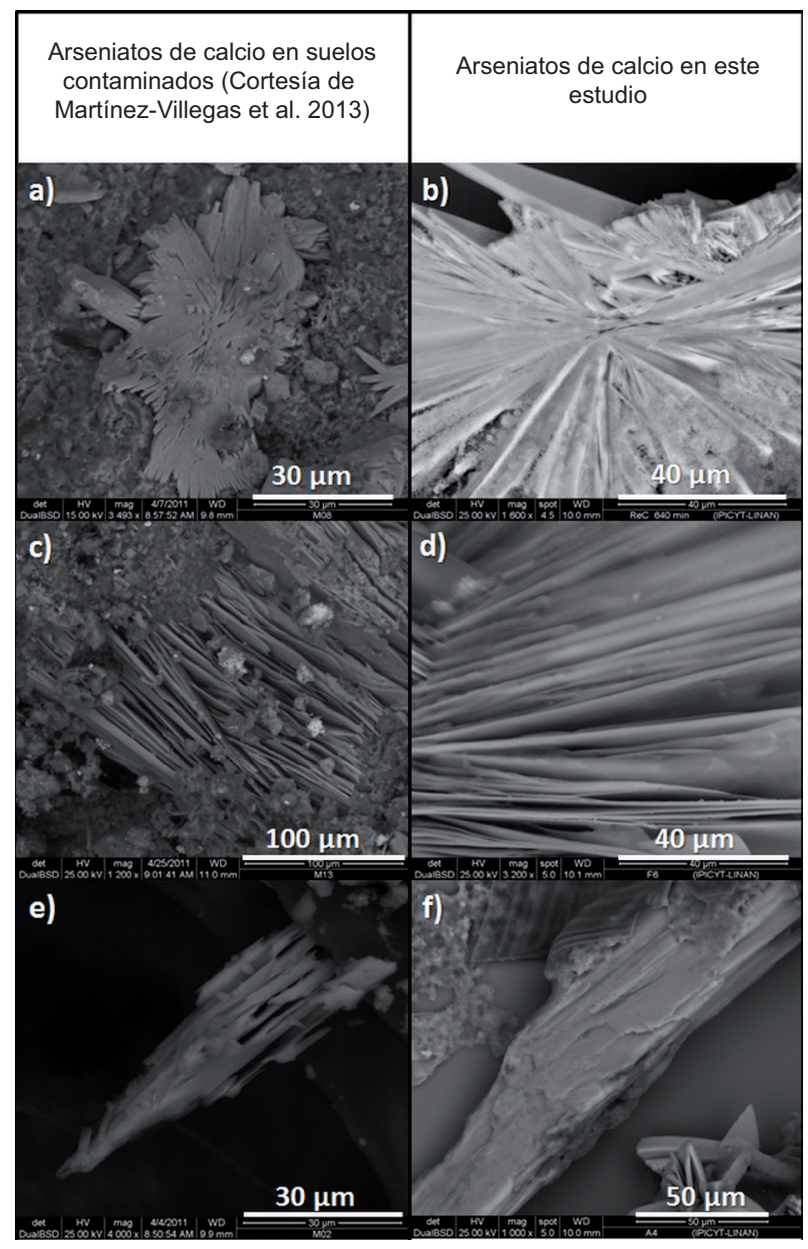

Fig. 8. Similitud entre las morfologías de arseniatos de calcio de muestras de suelos contaminados de Matehuala recolectados durante el estudio de Martínez-Villegas et al. (2013) y las morfologías de haidingerita, guerinita y farmacolita precipitadas en este estudio: a) arseniato de calcio de suelo contaminado con morfología similar a b) haidingerita; c) arseniato de calcio de suelo contaminado con morfología similar a d) guerinita; e) arseniato de calcio de suelo contaminado morfológicamente similar a f) farmacolita

La obtención de distintas fases de arseniatos de calcio en la síntesis 1 y 2 podría deberse a las diferencias de $\mathrm{pH}$ al comienzo de los experimentos (Fig. 1b). El pH es una variable maestra que actúa directamente sobre las especies acuosas de arsénico en solución $\left(\mathrm{H}_{3} \mathrm{AsO}_{4}, \mathrm{H}_{2} \mathrm{AsO}_{4}{ }^{-}, \mathrm{HAsO}_{4}{ }^{2-}, \mathrm{AsO}_{4}{ }^{3-}\right) \mathrm{y}$ la disponibilidad de calcio (en solución) proveniente de la disolución de $\mathrm{Ca}(\mathrm{OH})_{2}$. En las figuras $\mathbf{1 b}$ y 1d, se observa claramente que a menor $\mathrm{pH}$, mayor disponibilidad de calcio en la solución. Por otro lado, si bien dicha disponibilidad es indispensable para el proceso de precipitación, ésta no parece tener un efecto muy evidente en la precipitación del arsénico toda vez que la concentración de arsénico en la solución en el equilibrio fue similar en ambas síntesis (Fig. 1c). De acuerdo con la literatura, la precipitación de guerinita, haidingerita y farmacolita ocurre a través de las reacciones mostradas en el cuadro I.

Dentro de las mezclas de precipitados de guerinita, haidingerita y farmacolita obtenidas en este estudio se observaron los hábitos cristalinos característicos de cada una de las fases. La asociación de hábitos cristalinos con su respectiva fase mineral, representa una alternativa para el reconocimiento de arseniatos de calcio en muestras de suelos contaminados en el ambiente. Es decir, la similitud de los hábitos cristalinos observados en estudio y los observados en muestras de suelo contaminado en Matehuala sugiere que la presencia de guerinita, haidingerita y farmacolita es factible de encontrarse en las muestras de Matehuala. A la fecha, la presencia de $\mathrm{Ca}_{5} \mathrm{H}_{2}\left(\mathrm{AsO}_{4}\right)_{4}$. $n \mathrm{H}_{2} \mathrm{O}$ ha sido sugerida como el arseniato de calcio que controla la movilidad de arsénico en el acuífero de Matehuala y Cerrito Blanco (Martínez-Villegas et al. 2013). No obstante, la identificación inequívoca de éste (u otros arseniatos de calcio) no ha sido posible de corroborar debido a la posible presencia de variados y diferentes arseniatos de calcio en matrices compuestas principalmente por otros minerales primarios, tales como la calcita y el yeso (MartínezVillegas et al. 2013). La precipitación de guerinita y el reconocimiento de partículas con su morfología en suelos de Matehuala apoyan resultados previos de modelación hidrogeoquímica sobre la hipótesis de que un arseniato de calcio con esa estequiometría es el que controla la movilidad de arsénico en Matehuala. No obstante, estudios adicionales son necesarios para su identificación inequívoca y para avanzar en

CUADRO I. ESTEQUIOMETRÍA Y REACCIONES DE DISOLUCIÓN DE MINERALES DE ARSENIATO DE CALCIO

\begin{tabular}{lllll}
\hline $\mathrm{Ca} /$ As & Nombre & Fórmula química & Reacción de precipitación & $\log K_{p s}$ \\
\hline 1 & Haidingerita & $\mathrm{CaHAsO}_{4} \bullet \mathrm{H}_{2} \mathrm{O}$ & $\mathrm{Ca}^{2+}+\mathrm{HAsO}_{4}{ }^{2-}+\mathrm{H}_{2} \mathrm{O} \leftrightarrow \mathrm{CaHAsO}_{4} \bullet \mathrm{H}_{2} \mathrm{O}$ & $4.79(1)$ \\
1 & Farmacolita & $\mathrm{CaHAsO}_{4} \cdot 2 \mathrm{H}_{2} \mathrm{O}$ & $\mathrm{Ca}^{2+}+\mathrm{H}_{2} \mathrm{AsO}_{4}+2 \mathrm{H}_{2} \mathrm{O} \leftrightarrow \mathrm{CaHAsO}_{4} \cdot 2 \mathrm{H}_{2} \mathrm{O}+\mathrm{H}^{+}$ & $4.68(2)$ \\
1.25 & Guerinita & $\mathrm{Ca}_{5} \mathrm{H}_{2}\left(\mathrm{AsO}_{4}\right)_{4} \bullet 9 \mathrm{H}_{2} \mathrm{O}$ & $5 \mathrm{Ca}^{2+}+2 \mathrm{HAsO}_{4}{ }^{2-}+2 \mathrm{AsO}_{4}{ }^{3-}+9 \mathrm{H}_{2} \mathrm{O} \leftrightarrow \mathrm{Ca}_{5} \mathrm{H}_{2}\left(\mathrm{AsO}_{4}\right)_{4} \bullet 9 \mathrm{H}_{2} \mathrm{O}$ & $30.69(1)$ \\
\hline
\end{tabular}

(1) Bothe y Brown (1999), (2) Rodríguez-Blanco et al. (2007) 
la comprensión de los procesos y las variables que controlan los equilibrios y solubilidad de arseniatos de calcio en Matehuala.

\section{CONCLUSIONES}

La mezcla de soluciones de calcio y arsénico con relaciones iniciales $1.0<\mathrm{Ca} / \mathrm{As}<1.25$, en un rango de $\mathrm{pH}$ alcalino a ligeramente acidificado, dio origen a la precipitación de una fase de arseniato diprotonado de calcio: $\mathrm{Ca}_{5} \mathrm{H}_{2}\left(\mathrm{AsO}_{4}\right)_{4} \cdot 9 \mathrm{H}_{2} \mathrm{O}$ (guerinita) y dos fases de arseniatos monoprotonados de calcio: $\mathrm{CaHAsO}_{4}$. $\mathrm{H}_{2} \mathrm{O}$ (haidingerita) y CaHAsO $\mathrm{O}_{4} \cdot 2 \mathrm{H}_{2} \mathrm{O}$ (farmacolita), según los resultados de difracción de rayos $\mathrm{X}$ y microscopia electrónica de barrido. La precipitación de $\mathrm{Ca}_{5} \mathrm{H}_{2}\left(\mathrm{AsO}_{4}\right)_{4} \cdot 9 \mathrm{H}_{2} \mathrm{O}$ y de $\mathrm{CaHAsO}_{4} \cdot \mathrm{H}_{2} \mathrm{O}$, se identificó a pH 6.67 y una relación $\mathrm{Ca} / \mathrm{As}=1.3$, en el equilibrio. La precipitación de $\mathrm{Ca}_{5} \mathrm{H}_{2}\left(\mathrm{AsO}_{4}\right)_{4} \cdot 9 \mathrm{H}_{2} \mathrm{O}$, $\mathrm{CaHAsO}_{4} \cdot \mathrm{H}_{2} \mathrm{O}$ y $\mathrm{CaHAsO}_{4} \cdot 2 \mathrm{H}_{2} \mathrm{O}$ se observó en muestras a $\mathrm{pH} 6.5$ y relación $\mathrm{Ca} / \mathrm{As}=1.13$, en el equilibrio.

En general, la morfología de los arseniatos de calcio sintetizados incluyó hábitos laminares y fibrosos en agregados radiales que se asociaron a haidingerita, hábitos laminares aplanados que se relacionaron con guerinita y formas aciculares pertenecientes a farmacolita. Aunque el presente trabajo se realizó en condiciones de laboratorio, y sólo pretende emular los procesos de movilidad y de retención de arsénico por precipitación de arseniatos de calcio en ambientes reales, presenta una alternativa indirecta para acercarse a la solución de interrogantes sobre la identidad de los arseniatos de calcio que se encuentran en ambientes contaminados. En este sentido, los resultados de este estudio aportan al reconocimiento de que haidingerita, guerinita y farmacolita podrían encontrarse en los suelos contaminados de Matehuala.

\section{AGRADECIMIENTOS}

Este trabajo fue financiado con fondos de los proyectos CB-2012-183025 (SEP-CONACyT) y S-2694 (IPICYT-Geociencias Aplicadas). Gerardo Hernández agradece al Consejo Nacional de Ciencia y Tecnología (CONACyT) por la beca otorgada durante la realización de esta investigación (Núm. de becario 20815). Se agradece particularmente a Denise Ramírez Bautista por la clasificación y procesamiento de las micrografías. A la I.Q. Ma. del Carmen Rocha Medina del Laboratorio Nacional de Biotecnología Agrícola, Médica y Ambiental
(LANBAMA) y a las M.C. Ana Iris Peña Maldonado y Beatriz Adriana Rivera Escoto del Laboratorio Nacional de Investigaciones en Nanociencias y Nanotecnología (LINAN) por las facilidades otorgadas para la realización de los análisis experimentales. Finalmente, se agradece a los dos revisores anónimos cuyas observaciones contribuyeron en la mejora del manuscrito.

\section{REFERENCIAS}

Bothe J. V. y Brown P. W. (2002). CaO-As2O5-H2O system at 23 degrees $+/-1$ degrees C. J. Am. Ceram. Soc. 85, 221-224.

Bothe J. V. y Brown P. W. (1999a). Arsenic immobilization by calcium arsenate formation. Environ. Sci. Technol. 33, 3806-3811. DOI: 10.1021/es980998m

Bothe J. V. y Brown P. W. (1999b). The stabilities of calcium arsenates at 23+/-1 o C. J. Hazard. Mater. 69, 197-207. DOI: 10.1016/s0304-3894(99)00105-3

Castillo F., Ávalos-Borja M., Jamieson H., Hernández-Bárcenas G. y Martínez-Villegas N. (2015). Identification of diagenetic calcium arsenates using synchrotronbased micro X-ray diffraction. Bol. Soc. Geológica Mex. 67, 479-491.

Donahue R. y Hendry M. J. (2003). Geochemistry of arsenic in uranium mine mill tailings, Saskatchewan, Canada. Appl. Geochem. 18, 1733-1750. DOI: 10.1016/S0883-2927(03)00106-9

Jiménez A., Rodríguez-Blanco J. D., Torre L., GarcíaGranda S. y Prieto M. (2006). Cristaloquímica y comportamiento de cristalización de arseniatos de calcio. Memorias. XXVI Reunión de la Sociedad Española de Mineralogía (SEM)/ XX Reunión de la Sociedad Española de Arcillas (SEA) 2006. Oviedo, España. 11 al 14 de septiembre, pp. 267-270.

Martínez-Villegas N., Briones-Gallardo R., Ramos-Leal J. A., Avalos-Borja M., Castañón-Sandoval A. D., RazoFlores E. y Villalobos M. (2013). Arsenic mobility controlled by solid calcium arsenates: A case study in Mexico showcasing a potentially widespread environmental problem. Environ. Pollut. 176, 114-122. DOI: 10.1016/j.envpol.2012.12.025

MinDat (2016a). Hudson Institute of Mineralogy. Haidingerite [en línea]. http://www.mindat.org/min-1797. html 01/15/16.

MinDat (2016b). Hudson Institute of Mineralogy. Guerinite [en línea]. http://www.mindat.org/gm/1767 $01 / 20 / 16$.

MinDat (2016c). Hudson Institute of Mineralogy. Pharmacolite [en línea]. http://www.mindat.org/gm/3184 01/20/16. 
Myneni S. C. B., Traina S. J., Logan T.J. y Waychunas G. A. (1997). Oxyanion behavior in alkaline environments: Sorption and desorption of arsenate in ettringite. Environ. Sci. Technol. 31, 1761-1768. DOI:10.1021/es9607594

Nishimura T. y Robins R. G. (1998). A re-evaluation of the solubility and stability regions of calcium arsenites and calcium arsenates in aqueous solution at $25 \mathrm{oC}$. Miner. Process. Extr. Metall. Rev. Int. J. 18, 283-308. DOI: 10.1080/08827509808914159

Onac B. P., Hess J. W. y White W. B. (2007). The relationship between the mineral composition of speleothems and mineralization of breccia pipes: Evidence from corkscrew cave, Arizona, USA. Can. Mineral. 45, 1177-1188. DOI: 10.2113/gscanmin.45.5.1177

Ondrus P., Veselovsky F., Hlousek J., Skala R., Vavrin I., Fryda J., Cejka J. y Gabasova A. (1997). Secondary minerals of the Jachymov (Joachimsthal) ore district. J. Geosci. 42, 3-76.

Pantuzzo F. L. y Ciminelli V. S. T. (2010). Arsenic association and stability in long-term disposed arsenic residues. Water Res. 44, 5631-5640. DOI: 10.1016/j. watres.2010.07.011

Ravenscroft P., Brammer H. y Richards K. (2009). Arsenic pollution: A global synthesis. John Wiley and Sons, Oxford, Reino Unido, 588 pp.
Rodríguez-Blanco J. D., JiménezA. y Prieto M. (2007). Oriented overgrowth of pharmacolite $(\mathrm{CaHAsO} 4 \cdot 2 \mathrm{H} 2 \mathrm{O})$ on Gypsum (CaSO4-2H2O). Cryst. Growth Des. 7, 2756-2763.

DOI: $10.1021 / \operatorname{cg} 070222+$

Rodríguez J. D., Jiménez A., Prieto M., Torre P. y GarcíaGranda S. (2008). Interaction of gypsum with $\mathrm{As}(\mathrm{V})$ bearing aqueous solutions: Surface precipitation of guerinite, sainfeldite, and $\mathrm{Ca} 2 \mathrm{NaH}(\mathrm{AsO} 4)(2)$ center dot $6 \mathrm{H}(2) \mathrm{O}$, a synthetic arsenate. Am. Mineral. 93, 928-939. DOI: 10.2138/am.2008.2750

Schorn S. (2006). Mineralienatlas - Fossilienatlas. Guérinite [en línea]. http://www.mineralienatlas.de/index. php?lang=en\&language $=$ english 13/01/16

Smedley P. L. y Kinniburgh D. G. (2002). A review of the source, behaviour and distribution of arsenic in natural waters. Appl. Geochem. 17, 517-568. DOI: 10.1016/ S0883-2927(02)00018-5

Zhu Y. N., Zhang X. H., Xie Q. L., Wang D. Q. y Cheng G. W. (2006). Solubility and stability of calcium arsenates at 25o C. Water. Air. Soil Pollut. 169, 221-238. DOI: 10.1007/s11270-006-2099-y 